\title{
Fluctuation properties of the strength function associated with the giant quadrupole resonance in ${ }^{208} \mathrm{~Pb}$
}

\author{
Hirokazu Aiba \\ Kyoto Koka Women's College, 38 Kadono-cho Nishikyogoku, Ukyo-ku, 615-0882 Kyoto, Japan \\ Masayuki Matsuo \\ Department of Physics, Faculty of Science, Niigata University, 950-2181 Niigata, Japan \\ Shigeru Nishizaki \\ Faculty of Humanities and Social Sciences, Iwate University, 3-18-34 Ueda, 020-8550 Morioka, Japan \\ Toru Suzuki \\ Department of Physics, Tokyo Metropolitan University, 192-0397 Hachioji, Japan \\ (Received 25 June 2009; revised manuscript received 19 January 2011; published 23 February 2011)
}

\begin{abstract}
We performed fluctuation analysis by means of the local scaling dimension for the strength function of the isoscalar (IS) giant quadrupole resonance (GQR) in ${ }^{208} \mathrm{~Pb}$ where the strength function is obtained by the shell model calculation including $1 \mathrm{p} 1 \mathrm{~h}$ and $2 \mathrm{p} 2 \mathrm{~h}$ configurations. It is found that at almost all energy scales, fluctuation of the strength function obeys the Gaussian orthogonal ensemble (GOE) random matrix theory limit. This is contrasted with the results for the GQR in ${ }^{40} \mathrm{Ca}$, where at the intermediate energy scale of about $1.7 \mathrm{MeV}$, a deviation from the GOE limit was detected. It is found that the physical origin for this different behavior of the local scaling dimension is ascribed to the difference in the properties of the damping process.
\end{abstract}

DOI: 10.1103/PhysRevC.83.024314

PACS number(s): 24.60.Ky, 21.10.Pc, 24.30.Cz, 27.80.+w

\section{INTRODUCTION}

Giant resonances, excited by various probes, show, at an initial stage of the excitation process, a regular motion with a definite vibrational frequency [1,2]. These regular motions are then damped due to the coupling with a huge number of background states, and finally the so-called compound states are realized.

We now understand both ends of these processes: The frequency of the giant resonance, for instance, can be well evaluated by the random phase approximation (RPA). Compound states, on the other hand, are also well described by the random matrix theory with the Gaussian orthogonal ensemble (GOE) [3,4], which characterizes a classical chaotic motion.

It is still not well understood, however, how the dynamics changes from regular to chaotic [5]. To answer this question, it is very useful to study the fluctuation properties of the strength functions: The structure at the large energy scale of the strength function corresponds to the behavior of the initial stage, while the fluctuation properties at small energy scale correspond to the long time behavior.

We proposed and have used a novel fluctuation analysis based on the quantity we call the local scaling dimension to study the fluctuation properties of the strength functions [6]. This method is devised to quantitatively characterize the fluctuation at each energy scale, and is suitable for the investigation of the fine structure of the strength function.

The strength distribution of giant resonances and its fluctuation have also been studied experimentally. Recently, the fine structure of the strength distribution of the giant quadrupole resonance (GQR) in ${ }^{208} \mathrm{~Pb}$ [7-9] or the
Gamow-Teller resonance (GTR) in ${ }^{90} \mathrm{Zr}$ [10] were measured and theoretical analysis has also been done.

In the previous paper [11], we investigated the GQR in ${ }^{40} \mathrm{Ca}$, where the strength function was calculated by means of the second Tamm-Dancoff approximation (TDA), namely, the $1 \mathrm{p} 1 \mathrm{~h}$ and $2 \mathrm{p} 2 \mathrm{~h}$ model space is included. The results of the local scaling dimension analysis were as follows: At small energy scale, the behavior of the local scaling dimension is almost the same as that of the GOE, which exhibits the complexity of $2 \mathrm{p} 2 \mathrm{~h}$ background states. On the other hand, a clear deviation from the GOE was found at the intermediate energy scale, and it was found that this energy corresponds to the spreading width of $1 \mathrm{p} 1 \mathrm{~h}$ states. Hence, we can say that the spreading width of $1 \mathrm{p} 1 \mathrm{~h}$ states is detected as a deviation from the GOE limit in ${ }^{40} \mathrm{Ca}$.

For ${ }^{40} \mathrm{Ca}$, the fragmentation within $1 \mathrm{p} 1 \mathrm{~h}$ states is important for the damping process of the giant resonance. Namely, the strength is first fragmented over a wide range of $1 \mathrm{p} 1 \mathrm{~h}$ states, and this fragmentation characterizes a global profile of the total strength function. This fragmentation is often called Landau damping, although the original concept of Landau damping was considered for infinite systems. In this paper, we use the term Landau damping for fragmentation within $1 \mathrm{p} 1 \mathrm{~h}$ states for convenience.

However, as the mass of nuclei increases, the relative importance of Landau damping may change. Accordingly, $2 \mathrm{p} 2 \mathrm{~h}$ states may also contribute to the global profile of the strength function. Therefore, it is very important to investigate how the difference between the damping process of light nuclei and that of heavy nuclei does affect the properties of the fluctuation of the strength function. 
In this paper, we study the isoscalar (IS) GQR of ${ }^{208} \mathrm{~Pb}$, where the strength function is calculated with the second TDA in the same manner as in ${ }^{40} \mathrm{Ca}$, and we study the fluctuation of the strength function by means of the local scaling dimension. Comparing results with those for ${ }^{40} \mathrm{Ca}$, we would like to clarify which properties of the damping process are reflected in the fluctuation of the strength function and make clear the physical origin of the difference.

\section{GQR STRENGTH FUNCTION IN ${ }^{208}$ PB WITH COMPLEX 2p2h STATES}

\section{A. Microscopic strength function}

We calculate the strength function of the IS GQR in ${ }^{208} \mathrm{~Pb}$ within the second TDA including the $1 \mathrm{p} 1 \mathrm{~h}$ and $2 \mathrm{p} 2 \mathrm{~h}$ excitations. The adopted single-particle wave functions, singleparticle energies, and residual interaction are the same as those in Ref. [11]. The effective mass parameter $m^{*} / m$ is set to be 1 in this calculation. The model space was constructed in terms of single-particle states within the four major shells, two below and two above the Fermi surface. Then we included all $1 \mathrm{p} 1 \mathrm{~h}$ and $2 \mathrm{p} 2 \mathrm{~h}$ states whose unperturbed energies are less than $15 \mathrm{MeV}$. The resultant number of $1 \mathrm{p} 1 \mathrm{~h}$ and $2 \mathrm{p} 2 \mathrm{~h}$ states are 39 and 8032, respectively. We diagonalized the Hamiltonian within this model space and obtained the strength function

$$
S(E)=\sum_{i} S_{i} \delta\left(E-E_{i}+E_{0}\right),
$$

where $E_{i}$ and $E_{0}$ are energy eigenvalues, and $S_{i}$ is the isoscalar quadrupole strength of state $i$.

Figure 1 shows the calculated strength function. The average of the excitation energy weighted by the strength is about $10.5 \mathrm{MeV}$, and the standard deviation around the average is about $2.6 \mathrm{MeV}$, where all levels are considered. The peak position lies at the same value as the average. These values are consistent with the $\left(p, p^{\prime}\right)$ experimental data [7]. Moreover, the agreement of the global shape with the experimental data is also good. The dotted curve in Fig. 1 represents the smooth strength function by means of the Strutinsky method [12] with the smoothing width $0.2 \mathrm{MeV}$. The value of the full width

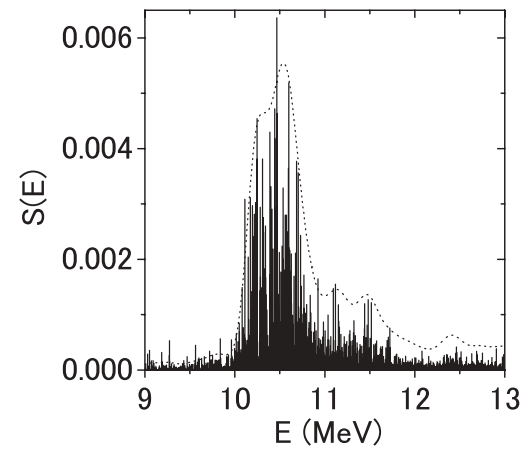

FIG. 1. Calculated strength function of the IS GQR in ${ }^{208} \mathrm{~Pb}$. Dotted curve shows the smooth strength function by means of the Strutinsky method with the smoothing width $0.2 \mathrm{MeV}$. A sum of strengths is normalized to unity. at half maximum (FWHM) of this smooth strength function is $0.63 \mathrm{MeV}$. To quantitatively characterize the spreading of the strength function around the largest peak, the FWHM is more appropriate than the standard deviation [13]. Thus, we use the FWHM as a measure of the total width $\Gamma$ of the strength function, which gives $\Gamma=0.63 \mathrm{MeV}$.

Hereafter, when we estimate the value of the FWHM, the same procedure as above is adopted, namely, we calculate the FWHM for the smooth strength function by means of the Strutinsky method with the smoothing width $0.2 \mathrm{MeV}$.

\section{B. Fluctuation at small scale}

To show the statistical properties of the levels and the strengths at small energy scales, we show here the nearestneighbor level spacing distribution (NND), the strength distribution, and $\Delta_{3}$ statistics. Here, the NND and strength distribution are measures characterizing the fluctuation at the small energy scale limit.

Figure 2(a) shows the NND. The NND follows the Wigner distribution well. We present the strength distribution in Fig. 2(b) where a histogram of the square root of normalized strengths is plotted. We also find that the distribution follows the Porter-Thomas one rather well. These two figures indicate that the fluctuation of the strength as well as that of the energy

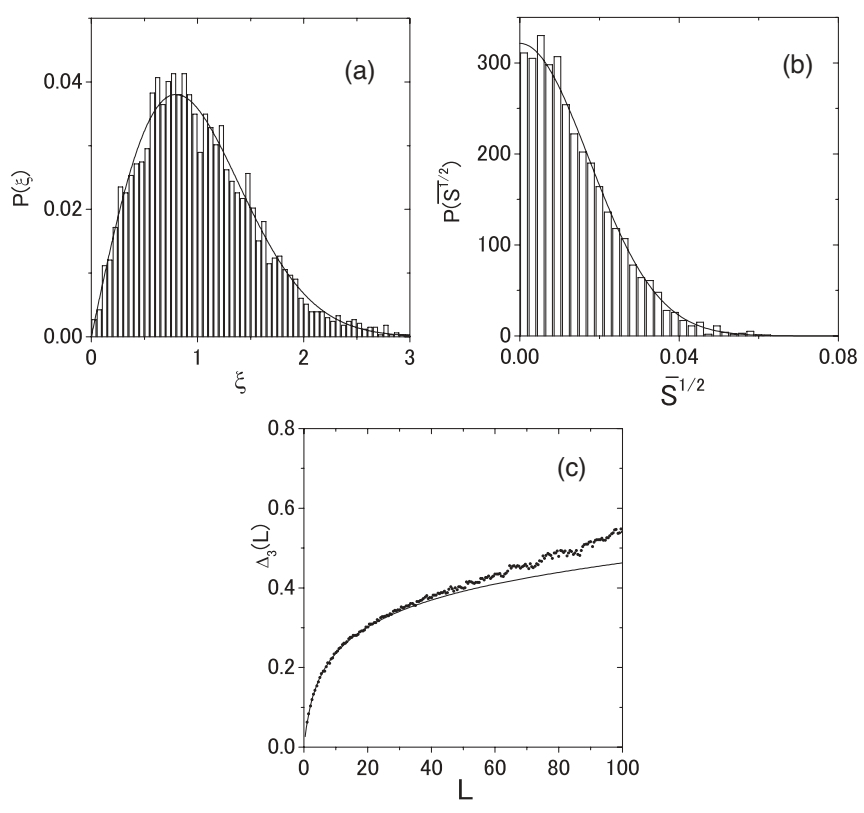

FIG. 2. (a) Nearest-neighbor level spacing distribution for ${ }^{208} \mathrm{~Pb}$. $\xi$ in the horizontal axis represents the spacing of unfolded levels. The solid curve represents the Wigner distribution. Level spacings were unfolded by the Strutinsky method with a smoothing width $0.5 \mathrm{MeV}$. (b) Histogram of the square root of normalized strengths $\bar{S}_{i}^{1 / 2}$ associated with IS GQR. The solid curve represents the PorterThomas distribution which becomes a Gaussian when plotted as a function of $\bar{S}_{i}^{1 / 2}$. See Sec. II C for the normalization of the strengths. (c) $\Delta_{3}$ statistics. The horizontal axis $L$ shows the value of the energy interval for the unfolded spectrum. The solid curve represents $\Delta_{3}$ for the GOE level fluctuation. For each panel, 3321 levels between 9.9 and $13.1 \mathrm{MeV}$ are considered. 
level spacing is governed by the GOE at least at small energy scale limit.

Figure 2(c) shows the $\Delta_{3}$ statistics. We again find that at small energy range, the $\Delta_{3}$ follows the GOE line, although at intermediate energy scales, $L_{\max } \simeq 20$, the $\Delta_{3}$ starts to deviate from the GOE line to upward.

The GOE fluctuations at small energy scales are in accordance with the case of ${ }^{40} \mathrm{Ca}[11,14]$.

\section{Local scaling dimension}

Energy-scale dependence of the strength fluctuations can be measured in terms of the local scaling dimension $[6,11]$. In evaluating this quantity, we normalize the individual strength as $\bar{S}_{i}=S_{i} / f\left(E_{i}\right)$, where $f(E) \propto \tilde{S}(E) / \tilde{\rho}(E)$ is a smooth function of $E$, so that we remove effects of the global shape of the strength function $\tilde{S}(E)$ and the level density $\tilde{\rho}(E)$. We obtain $\tilde{S}(E)$ by convoluting the original $S(E)$ with a smoothing function of the Strutinsky type, for which we adopt $0.5 \mathrm{MeV}$ as the value of the width parameter $\omega$. The local scaling dimension as a function of energy scale $\epsilon$ is given as

$$
D_{m}(\epsilon) \equiv \frac{1}{m-1} \frac{\partial \log \chi_{m}(\epsilon)}{\partial \log \epsilon},
$$

where $\chi_{m}(\epsilon) \equiv \sum_{n=1}^{N} p_{n}^{m}$ is the $m$ th moment, called the partition function, of the fluctuation in the binned strengths $p_{n}$, which are sums of the strength $\bar{S}_{i}$ in energy bins with the energy interval $\epsilon$. For details, we refer the reader to Refs. [6,11]. We analyze $33212^{+}$levels in the energy range from 9.9 to 13.1 MeV.

Figures 3(a) and 3(b) represent the partition function and the local scaling dimension, respectively, of IS GQR in ${ }^{208} \mathrm{~Pb}$. The horizontal axes in both figures represent the bin width $\epsilon$ of energy in unit of $d$, where $d$ represents the average level spacing over the energy range $9.9-13.1 \mathrm{MeV}(d=0.96 \mathrm{keV})$. The partition function clearly deviates from the linear relation in the log-log plot. This means that for the IS GQR strength function, the self-similar property does not hold. We can also see a more detailed structure in the figure of the local scaling dimension. At the smallest energy scale $\epsilon \simeq d$, the value of the local scaling dimension is small, $D_{2} \simeq 0.35$, which means that the fluctuation is very large at small energy scales. As
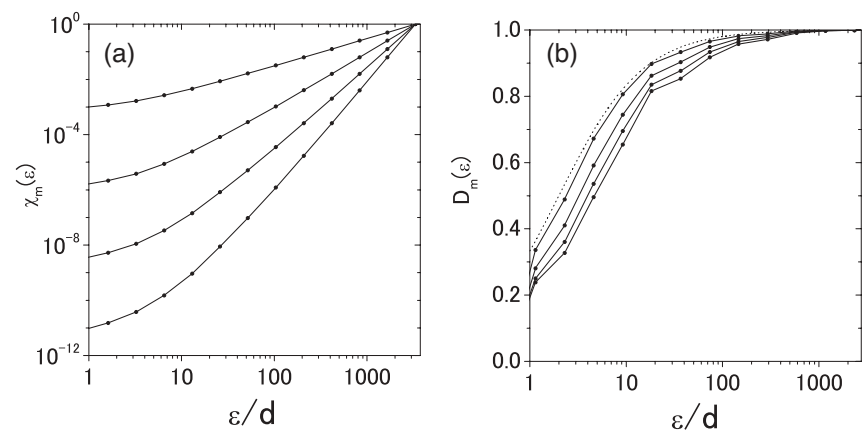

FIG. 3. Partition function (a) and local scaling dimension (b) for the IS GQR in ${ }^{208} \mathrm{~Pb}$. Curves in each figure correspond to $m=2-$ 5 from upper to lower. Dotted curves in (b) represent $D_{2}(\epsilon)$ for the GOE. the energy scale or the bin width increases, the values of $D_{m}(\epsilon)$ monotonically increase. Finally, at about $\epsilon \simeq 100 d$ the values of $D_{m}(\epsilon)$ converge to unity, which indicates that at large energy scales, the strength function appears smooth. The most important feature in Fig. 3(b) is that the local scaling dimension for ${ }^{208} \mathrm{~Pb}$ almost follows the GOE line at almost all the energy scales.

This should be contrasted with the case of ${ }^{40} \mathrm{Ca}[11]$ : When the energy scale is small, the local scaling dimension almost follows the GOE line. As the energy scale increases, however, we can find a dip and a deviation from the GOE line at about 1.7 $\mathrm{MeV}$, which is approximately related to the value of the spreading width of $1 \mathrm{p} 1 \mathrm{~h}$ states.

Note that if we look only at the small or large energy scale limits, we cannot find the difference between ${ }^{208} \mathrm{~Pb}$ and ${ }^{40} \mathrm{Ca}$. Studies of fluctuation at intermediate energy scales lead to the finding of the difference. In the following we shall investigate the mechanism which brings about the difference in fluctuations at intermediate energy scales.

\section{ANALYSIS OF DAMPING PROCESS}

Let us now investigate origins of the difference between the cases of ${ }^{40} \mathrm{Ca}$ and ${ }^{208} \mathrm{~Pb}$. In our previous study of the GQR in ${ }^{40} \mathrm{Ca}$, we showed that the behavior of the local scaling dimension can be interpreted in terms of the doorway damping mechanism. We here employ the same picture in order to clarify the damping mechanism of the GQR in ${ }^{208} \mathrm{~Pb}$.

The doorway damping mechanism consists of a two-step process which is illustrated in Fig. 4. The giant resonance is spread over the $1 \mathrm{p} 1 \mathrm{~h}$ states due to Landau damping, the width of which is denoted by $\Gamma_{\mathrm{L}}$. The average spacing of $1 \mathrm{p} 1 \mathrm{~h}$ states is denoted by $D_{1 \mathrm{p} 1 \mathrm{~h}}$. The $1 \mathrm{p} 1 \mathrm{~h}$ states are considered here as the "doorway" states of the damping process. The $1 \mathrm{p} 1 \mathrm{~h}$ states then couple to more complicated background states $(2 \mathrm{p} 2 \mathrm{~h}$ states) through the residual two-body interaction. The coupling causes the spreading width of $1 \mathrm{p} 1 \mathrm{~h}$ states, which we denote $\gamma_{12}$. We define the "GQR TD" state as the Tamm-Dancoff (TD) state with the largest quadrupole strength among all TD states, where the "TD" states mean the states obtained in the TDA, i.e., by the diagonalization within the model space limited to the $1 \mathrm{p} 1 \mathrm{~h}$ configurations. The GQR TD state also couples to $2 \mathrm{p} 2 \mathrm{~h}$ states, and hence it should have the spreading width due to the coupling. This is similar to $\gamma_{12}$, but we introduce a separate symbol $\Gamma_{2}$ since the GQR TD state is a special state consisting of a coherent superposition

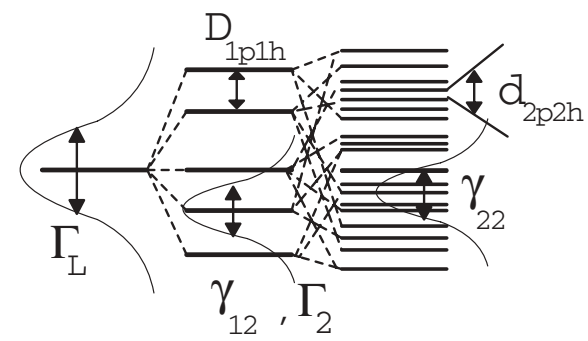

FIG. 4. Schematic drawing of the doorway damping mechanism of the giant resonance, and related quantities. 
of many unperturbed $1 \mathrm{p} 1 \mathrm{~h}$ excitations. $d_{2 \mathrm{p} 2 \mathrm{~h}}$ is the average spacing of background $2 \mathrm{p} 2 \mathrm{~h}$ states. The residual interaction also acts among the $2 \mathrm{p} 2 \mathrm{~h}$ states, and the mixing among the $2 \mathrm{p} 2 \mathrm{~h}$ states causes a spreading width of the $2 \mathrm{p} 2 \mathrm{~h}$ states, which we denote $\gamma_{22}$.

In the following we shall evaluate all these quantities in order to clarify the damping mechanism of the GQR in ${ }^{208} \mathrm{~Pb}$ (Secs. III A and III B). We also study whether there are specific states among 2p2h states which strongly couple with the GQR mode (Sec. III C) and then discuss the difference of the nature associated with the fluctuation of the strength function between ${ }^{40} \mathrm{Ca}$ and ${ }^{208} \mathrm{~Pb}$ (Sec. III D).

\section{A. Mechanism producing the total width}

\section{Landau damping}

For ${ }^{40} \mathrm{Ca}$, Landau damping is important, so the strengths are already fragmented in the $1 \mathrm{p} 1 \mathrm{~h}$ levels. Therefore we first would like to investigate in ${ }^{208} \mathrm{~Pb}$ how the strength is distributed in the TDA where only the $1 \mathrm{p} 1 \mathrm{~h}$ states are included.

Figure 5(a) shows the TDA strength function, which is obtained by means of the TDA, namely, by neglecting $2 \mathrm{p} 2 \mathrm{~h}$ states, of the IS quadrupole operator. Different from the case of ${ }^{40} \mathrm{Ca}$, strengths in the GQR region are considerably concentrated on the single peak located at about $10.7 \mathrm{MeV}$. Because of this, the TDA strength function is very different from the full strength function in Fig. 1. At the same time, we also see only a small effect of Landau damping. In fact, the strength concentration on the single peak at $E=10.7 \mathrm{MeV}$ is $59 \%$ of the strengths in the energy interval $9-13 \mathrm{MeV}$. The Landau damping width $\Gamma_{\mathrm{L}}$ may be evaluated in terms of a smoothed profile of the strength function plotted with the dotted curve in Fig. 5(a). Its FWHM reads $0.21 \mathrm{MeV}$. On the other hand, if we closely look at Fig. 5(a), we find that there is the second largest peak just below the largest one and that these two levels dominate the whole structure. The level spacing between these two levels can be considered as a typical spreading of strength and may be a more direct quantitative measure of the Landau damping width $\Gamma_{\mathrm{L}}$ : The level spacing $0.18 \mathrm{MeV}$ gives $\Gamma_{\mathrm{L}}=0.18 \mathrm{MeV}$.
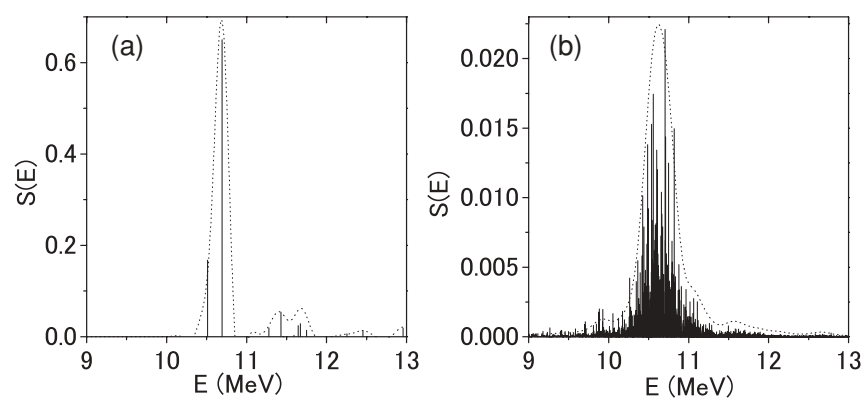

FIG. 5. (a) TDA strength function for the IS quadrupole operator in ${ }^{208} \mathrm{~Pb}$. (b) Strength function by neglecting all TD states except the GQR TD state. A total of 3342 2p2h states lying in 9-13 MeV are considered. See Fig. 1 for the dotted curve.

\section{Damping due to $2 p 2 h$ states}

The Landau damping width $\Gamma_{\mathrm{L}}=0.18 \mathrm{MeV}$ is not enough to explain the total width $\Gamma=0.63 \mathrm{MeV}$ of Sec. II. Then, we would like to study a role of $2 \mathrm{p} 2 \mathrm{~h}$ states in the damping process, namely, the fragmentation of the GQR TD state located at $E=$ 10.7 MeV in Fig. 5(a) over 2p2h states. We shall investigate the damping width $\Gamma_{2}$ caused by the coupling to $2 \mathrm{p} 2 \mathrm{~h}$ states. To estimate this width, we perform a calculation where we include only the GQR TD state and $2 \mathrm{p} 2 \mathrm{~h}$ states, where the coupling between the GQR TD state and $2 \mathrm{p} 2 \mathrm{~h}$ states as well as the interaction among $2 \mathrm{p} 2 \mathrm{~h}$ states is taken into account.

Figure 5(b) shows the resulting strength function. The estimated FWHM is $0.41 \mathrm{MeV}$, i.e., $\Gamma_{2}=0.41 \mathrm{MeV}$.

If the Landau damping and the $2 \mathrm{p} 2 \mathrm{~h}$ damping are independent of each other, and neighboring TD states around the GQR TD states also have the same spreading width as $\Gamma_{2}$, the following approximate relation holds:

$$
\Gamma \simeq \Gamma_{\mathrm{L}}+\Gamma_{2} \text {. }
$$

The values, $\Gamma_{\mathrm{L}}=0.18 \mathrm{MeV}$ and $\Gamma_{2}=0.41 \mathrm{MeV}$, estimated above, indeed satisfy this relation. Consequently, the total width $\Gamma=0.63 \mathrm{MeV}$ is approximately explained as a sum of the Landau damping width $\Gamma_{L}$ and the $2 \mathrm{p} 2 \mathrm{~h}$ damping width $\Gamma_{2}$.

\section{B. Spreading width of $1 \mathrm{p} 1 \mathrm{~h}$ states and $2 \mathrm{p} 2 \mathrm{~h}$ states}

Let us evaluate the spreading width $\gamma_{12}$ of the $1 \mathrm{p} 1 \mathrm{~h}$ states due to the coupling with $2 \mathrm{p} 2 \mathrm{~h}$ states. We shall also evaluate the spreading width $\gamma_{22}$ of $2 \mathrm{p} 2 \mathrm{~h}$ states, which is caused by the residual coupling among $2 \mathrm{p} 2 \mathrm{~h}$ states.

We evaluate $\gamma_{12}$ by using the strength functions of TD states as in Ref. [11]. Namely, we calculate the strength function of each TD state. Averaging the strength functions over whole TD states, we obtain Fig. 6(a). The FWHM of this averaged strength function gives an evaluation of the spreading width $\gamma_{12}$. We read $\gamma_{12}=0.38 \mathrm{MeV}$. (Note that we define $\gamma_{12}$ as the spreading width of TD states instead of that of unperturbed $1 \mathrm{p} 1 \mathrm{~h}$ states.) The value of spreading width of $2 \mathrm{p} 2 \mathrm{~h}$ states $\gamma_{22}$ is also evaluated in the same manner. From Fig. 6(b), we also obtain $\gamma_{22}=0.75 \mathrm{MeV}$ as the estimate of the spreading width of $2 \mathrm{p} 2 \mathrm{~h}$ states. These results will be used in Sec. III D.

For the sake of comparison, let us estimate the spreading width by assuming the Fermi golden rule. The root mean square of matrix elements between $1 \mathrm{p} 1 \mathrm{~h}$ states and $2 \mathrm{p} 2 \mathrm{~h}$ states is calculated as
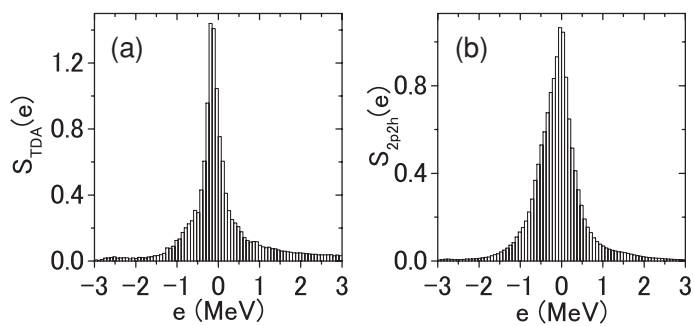

FIG. 6. Averaged strength function of (a) TD states and (b) $2 \mathrm{p} 2 \mathrm{~h}$ states. Average was performed over levels lying in 9-13 MeV. The number of levels is 12 and 3342 for TD states and $2 \mathrm{p} 2 \mathrm{~h}$ states, respectively. 
$\left(\overline{\left\langle 1 \mathrm{p} 1 \mathrm{~h}\left|V_{12}\right| 2 \mathrm{p} 2 \mathrm{~h}\right\rangle^{2}}\right)^{1 / 2}=9.3 \times 10^{-3} \mathrm{MeV} . \quad$ Similarly, we calculate $\left(\overline{\left\langle 2 \mathrm{p} 2 \mathrm{~h}\left|V_{22}\right| 2 \mathrm{p}^{\prime} 2 \mathrm{~h}^{\prime}\right\rangle^{2}}\right)^{1 / 2}=1.0 \times 10^{-2} \mathrm{MeV}$. Since the level spacing of $2 \mathrm{p} 2 \mathrm{~h}$ states is $d_{2 \mathrm{p} 2 \mathrm{~h}}=1.2 \mathrm{keV}$, the spreading widths $\gamma_{12}$ and $\gamma_{22}$ are approximately estimated in the Fermi golden rule as $\gamma_{12}^{\mathrm{FG}}=2 \pi \overline{\left\langle 1 \mathrm{p} 1 \mathrm{~h}\left|V_{12}\right| 2 \mathrm{p} 2 \mathrm{~h}\right\rangle^{2}} / d_{2 \mathrm{p} 2 \mathrm{~h}}=$ $0.46 \mathrm{MeV} \quad$ and $\quad \gamma_{22}^{\mathrm{FG}}=2 \pi \overline{\left\langle 2 \mathrm{p} 2 \mathrm{~h}\left|V_{22}\right| 2 \mathrm{p}^{\prime} 2 \mathrm{~h}^{\prime}\right\rangle^{2}} / d_{2 \mathrm{p} 2 \mathrm{~h}}=$ $0.53 \mathrm{MeV}$, respectively, which are in approximate agreement with the direct evaluation within $30 \%$.

\section{Search for strongly coupled states in $2 \mathrm{p} 2 \mathrm{~h}$ states}

In Fig. 4, 2p2h states are assumed to play a role as the chaotic background and provide the GOE fluctuation to the strength function. However, if the GQR TD state couples with not all $2 \mathrm{p} 2 \mathrm{~h}$ states equally but specific states in $2 \mathrm{p} 2 \mathrm{~h}$ states strongly, there is a possibility for this hierarchical structure in $2 \mathrm{p} 2 \mathrm{~h}$ states to give rise to a deviation from the GOE fluctuation. We, here, would like to investigate whether whole $2 \mathrm{p} 2 \mathrm{~h}$ states are rather equally coupled with the GQR TD state or whether there are specific states in $2 \mathrm{p} 2 \mathrm{~h}$ states which strongly couple with that state.

As a candidate of such specific states, we can consider the low-energy surface vibration plus $1 \mathrm{p} 1 \mathrm{~h}$ states. In Refs. [13,15-18], the importance of the coupling to the surface vibration in the wide range of damping phenomena including the damping of a single-particle motion as well as that of giant resonances was discussed. As for the giant resonance, which is composed of a coherent superposition of $1 \mathrm{p} 1 \mathrm{~h}$ states, this means that the damping occurs via the coupling with the specific $2 \mathrm{p} 2 \mathrm{~h}$ states, namely, the surface vibration plus $1 \mathrm{p} 1 \mathrm{~h}$ (s.v. $+1 \mathrm{p} 1 \mathrm{~h})$ states.

Since our model does not assume the particle-vibration coupling a priori, it is not trivial whether our model also has a mechanism that enhances the coupling with the lowenergy surface vibration. Therefore, we would like to study whether the s.v. $+1 \mathrm{p} 1 \mathrm{~h}$ states are particularly strongly coupled with the GQR TD state within our model. To do so, we calculate the FWHM of the following approximate strength function:

$$
S(E)=-\frac{1}{\pi} \operatorname{Im}\left(E-E_{c}-\sum_{\alpha} \frac{V_{c \alpha}^{2}}{E-\omega_{\alpha}+i \gamma_{22} / 2}\right)^{-1},
$$

where, $E_{c}$ and $\omega_{\alpha}$ denote the energy of the GQR TD state and the energy of the $\alpha$ th s.v. $+1 \mathrm{p} 1 \mathrm{~h}$ state, respectively. $V_{c \alpha}$ represents the coupling matrix element between the GQR TD state and the s.v. $+1 \mathrm{p} 1 \mathrm{~h}$ state $\alpha$.

Only $J^{\pi}=2^{+}, 3^{-}$modes are included as surface vibrations. We took only the lowest TD state as the $J^{\pi}=2^{+}$surface vibrational mode. On the other hand, we must pay attention to the collectivity of the octupole mode. The TDA strength function for the IS octupole operator shows that the energy of the lowest state is too high, and strengths are fragmented over several states compared with the experimental data [19]. Thus, we took into account the lowest nine states for the octupole mode. Note that s.v. $+1 \mathrm{p} 1 \mathrm{~h}$ states thus defined are not orthogonal. In this sense, Eq. (4) is an approximation which neglects the nonorthogonality.
TABLE I. Averaged value of squared coupling matrix elements $\overline{V_{c \alpha}^{2}}$ between the GQR TD state and surface vibration plus $1 \mathrm{p} 1 \mathrm{~h}$ states or the whole $2 \mathrm{p} 2 \mathrm{~h}$ states (third column), the associated spreading width $\Gamma_{2}^{\mathrm{FG}}$ of the GQR TD state evaluated by the Fermi golden rule (fourth column), and the spreading width $\Gamma_{2}$ estimated by the FWHM of the strength function based on Eq. (4) (fifth column). Second column shows the number of states considered. The first row shows the results obtained by including only the s.v. $+1 \mathrm{p} 1 \mathrm{~h}$ states while the second row shows those for the case of the whole $2 \mathrm{p} 2 \mathrm{~h}$ states.

\begin{tabular}{lcccr}
\hline \hline & No. & $\overline{V_{c \alpha}^{2}}\left(\mathrm{MeV}^{2}\right)$ & $\Gamma_{2}^{\mathrm{FG}}(\mathrm{MeV})$ & $\Gamma_{2}(\mathrm{MeV})$ \\
\hline S.v. $+1 \mathrm{p} 1 \mathrm{~h}$ & 909 & $0.65 \times 10^{-4}$ & 0.092 & 0.074 \\
2p2h & 3342 & $0.72 \times 10^{-4}$ & 0.38 & 0.41 \\
\hline \hline
\end{tabular}

We calculated the strength function based on Eq. (4) $\left(\gamma_{22}=\right.$ $0.75 \mathrm{MeV}$ was used). Then, the width $\Gamma_{2}^{\text {(s.v.) }}$ estimated by the FWHM was $0.074 \mathrm{MeV}$. This value is significantly smaller than the width $\Gamma_{2}=0.41 \mathrm{MeV}$ of the GQR TD state caused by the coupling to the whole $2 \mathrm{p} 2 \mathrm{~h}$ states.

From the estimate by the Fermi golden rule, we can give more detailed comparison between the width for the case of s.v. $+1 \mathrm{p} 1 \mathrm{~h}$ states and that for the whole $2 \mathrm{p} 2 \mathrm{~h}$ states. It is noted in Table I that the spreading width $\Gamma_{2}^{\text {(s.v. })}=0.074 \mathrm{MeV}$ and $\Gamma_{2}=0.41 \mathrm{MeV}$ are well accounted for by the estimate. In the Fermi golden rule, the spreading width is governed by two factors: (1) the average value of squared coupling matrix elements $\overline{V_{c \alpha}^{2}}$ between the GQR TD state and the states that couple to it, and (2) the level density of the coupling states. From Table I, we see that the large difference between the two widths simply reflects the difference between the number of s.v. $+1 \mathrm{p} 1 \mathrm{~h}$ states (909) and 2p2h states (3342), whereas the coupling strength of s.v. $+1 \mathrm{p} 1 \mathrm{~h}$ states $\overline{V_{c \alpha}^{2}}=0.65 \times$ $10^{-4} \mathrm{MeV}^{2}$ is comparable to the coupling strength $\overline{V_{c \alpha}^{2}}=$ $0.72 \times 10^{-4} \mathrm{MeV}^{2}$ for the whole $2 \mathrm{p} 2 \mathrm{~h}$ states.

Table I and the strength function based on Eq. (4) suggest that our model does not contain the enhancement of the coupling with the surface vibrations in the damping of the GQR. Therefore we consider in the following the $2 \mathrm{p} 2 \mathrm{~h}$ states as background states which do not have specific structures.

\section{Physical origin of the difference between ${ }^{40} \mathrm{Ca}$ and ${ }^{208} \mathrm{~Pb}$}

In the above sections, we evaluated the physical quantities such as the various spreading widths, with which we discussed the damping process, especially the mechanism of producing the total width of the strength function. Here, using these quantities we would like to discuss the physical origin of the difference between the fluctuation of the strength fluctuation of ${ }^{40} \mathrm{Ca}$ and that of ${ }^{208} \mathrm{~Pb}$. Table II summarizes the values of

TABLE II. Values of physical quantities related to the damping of the GQR for ${ }^{40} \mathrm{Ca}$ and ${ }^{208} \mathrm{~Pb}$. Unit of energy is keV for all cases.

\begin{tabular}{rrrrrrrr}
\hline \hline & \multicolumn{1}{c}{$\Gamma$} & \multicolumn{1}{c}{$\Gamma_{L}$} & \multicolumn{1}{c}{$\Gamma_{2}$} & $\gamma_{12}$ & $D_{1 \mathrm{plh}}$ & $\gamma_{22}$ & $d_{2 \mathrm{p} 2 \mathrm{~h}}$ \\
\hline${ }^{40} \mathrm{Ca}$ & 4100 & 2100 & 1500 & 1500 & 500 & 5200 & 11 \\
${ }^{208} \mathrm{~Pb}$ & 630 & 180 & 410 & 380 & 230 & 750 & 1.2 \\
\hline \hline
\end{tabular}


the above physical quantities related to the initial stage of the damping process for both ${ }^{40} \mathrm{Ca}$ and ${ }^{208} \mathrm{~Pb}$.

Here, we would like to mention the relation between $\Gamma$ and $\gamma_{22}$. One may wonder how the spreading width $\gamma_{22}$ affects the total width $\Gamma$ since the value of $\gamma_{22}$ is larger than that of $\Gamma$ for both nuclei. The relation can be understood from Eq. (4). From Eq. (4) we find that the spreading width $\gamma_{22}$ does not affect the total width $\Gamma$ but has a smoothing effect for the strength function.

We have shown in our previous study [6] that the damping process through the doorway states causes large fluctuations which have characteristic energy scales, and that the fluctuations emerge in the local scaling dimension. For instance, the energy scale of the spreading width $\gamma_{12}$ of the doorway states is the quantity which shows up prior to the other quantities. It is noted, however, that the size of the fluctuations depends on the mutual relations among the quantities mentioned above.

Studying more quantitatively with the use of the doorway damping model of Ref. [6], we found the condition to detect the effect of the spreading width $\gamma_{12}$ as

(i) $\gamma_{12} \leqslant 4 D_{1 \mathrm{p} 1 \mathrm{~h}}$

Furthermore, we need the second condition

(ii) $\gamma_{12}<\Gamma_{L}$.

This simply means that the spreading width $\gamma_{12}$ of the doorway states (1p1h states) need to be smaller than the total width $\Gamma$. Since $\Gamma \simeq \Gamma_{L}+\Gamma_{2}$ and $\gamma_{12} \simeq \Gamma_{2}$, the requirement $\gamma_{12}<\Gamma$ can be written as (ii). In addition to (i) and (ii), we need the third condition

$$
\text { (iii) } D_{1 \mathrm{p} 1 \mathrm{~h}}<\Gamma_{L} \text {. }
$$

This is because we need more than one doorway state within the the energy interval $\Gamma_{L}$ in order to have fluctuating behavior in the strength function.

Let us first look at the case of ${ }^{40} \mathrm{Ca}$. From Table II, the relation $\gamma_{12}=3.0 D_{1 \mathrm{p} 1 \mathrm{~h}}$ is derived, and this relation fulfills condition (i). On the other hand, relations $\gamma_{12}=0.71 \Gamma_{L}$ and $D_{1 \mathrm{p} 1 \mathrm{~h}}=0.24 \Gamma_{L}$ are also derived from Table II, and these relations satisfy both conditions (ii) and (iii). As a result, for ${ }^{40} \mathrm{Ca}$, we can see a deviation from the GOE fluctuation in the local scaling dimension, and indeed the energy scale where the deviation is seen is related to the value of $\gamma_{12}$.

For ${ }^{208} \mathrm{~Pb}$, on the other hand, we find in Table II that $\gamma_{12}=1.7 D_{1 \mathrm{p} 1 \mathrm{~h}}$, while $\Gamma_{L}$ is smaller than $\gamma_{12}$ and $D_{1 \mathrm{p} 1 \mathrm{~h}}$, i.e., $\gamma_{12}=2.1 \Gamma_{L}$ and $D_{1 \mathrm{p} 1 \mathrm{~h}}=1.3 \Gamma_{L}$. The first relation satisfies condition (i). The latter two relations, however, break conditions (ii) and (iii). Accordingly, for ${ }^{208} \mathrm{~Pb}$, the deviation from the GOE due to the effect of $\gamma_{12}$ cannot be seen. The essential physica 1 origin of this difference is that for ${ }^{208} \mathrm{~Pb}$ the Landau damping width is small compared to that for ${ }^{40} \mathrm{Ca}$. The smallness or largeness of the value of the Landau damping width affects the fluctuation property of the strength function.

\section{CONCLUSION}

We studied the fluctuation properties of the strength function of IS GQR for ${ }^{208} \mathrm{~Pb}$ by means of the local scaling dimension, and compared the results with those of ${ }^{40} \mathrm{Ca}$. The strength function was obtained by the second TDA including $2 \mathrm{p} 2 \mathrm{~h}$ states as well as $1 \mathrm{p} 1 \mathrm{~h}$ states. For ${ }^{40} \mathrm{Ca}$, we find a fluctuation different from GOE around the energy scale which is approximately related to the spreading width of the $1 \mathrm{p} 1 \mathrm{~h}$ states. On the other hand, for ${ }^{208} \mathrm{~Pb}$ we cannot find the fluctuation different from the GOE at almost all the energy scales.

The different behavior of the fluctuation detected by the local scaling dimension analysis is due to the difference of the ratio of the Landau damping width $\Gamma_{L}$ to the spreading width of the $1 \mathrm{p} 1 \mathrm{~h}$ states $\gamma_{12}$.

Recently, the analysis of the strength function of the IS GQR in ${ }^{208} \mathrm{~Pb}$ obtained by a $\left(p, p^{\prime}\right)$ inelastic scattering experiment was performed by means of the wavelet transform [7]. The authors suggest from the positions of the local maxima in the wavelet power that there exist three energy scales in the fluctuation of the strength function: I. $120 \mathrm{keV}$, II. 440 and $850 \mathrm{keV}$, and III. $1500 \mathrm{keV}$. Existence of the higher two energy scales is not inconsistent with our results, since our analysis says nothing about the fluctuation at about energy scale II, which may correspond to the total width $\Gamma$ in our model, or higher energy scales. However, the existence of the smallest energy scale $\sim 120 \mathrm{keV}$ may conflict with our results. If there is such an energy scale in our strength function, our analysis must detect it as a deviation from the GOE fluctuation. Therefore, it is very important to study the origin of this discrepancy. In particular, it is interesting to clarify the relation between the two methods, namely, the local scaling dimension and the wavelet power. Studies in this direction are now in progress.

\section{ACKNOWLEDGMENTS}

The authors acknowledge helpful discussion with K. Matsuyanagi. We are also indebted to A. Richter and P. von Neumann-Cosel for many fruitful discussions. The numerical calculations were performed at the Yukawa Institute Computer Facility as well as at the RCNP Computer Facility.
[1] Electric and Magnetic Giant Resonances in Nuclei, edited by J. Speth (World Scientific, Singapore, 1991).

[2] M. N. Harakeh and A. van der Woude, Giant Resonances (Oxford University Press, Oxford, England, 2001).

[3] F. J. Dyson, J. Math. Phys. 3, 140 (1962); 3, 157, (1962); 3, 166 (1962).

[4] M. L. Mehta, Random Matrices, 2nd ed. (Academic, New York, 1991).
[5] B. Mottelson, in Trends in Nuclear Physics, 100 Years Later, Les Houches, Session LXVI, edited by H. Nifenecker et al. (Elsevier, New York, 1998).

[6] H. Aiba and M. Matsuo, Phys. Rev. C 60, 034307 (1999).

[7] A. Shevchenko et al., Phys. Rev. Lett. 93, 122501 (2004).

[8] A. Shevchenko et al., Phys. Rev. C 77, 024302 (2008).

[9] D. Lacroix and P. Chomaz, Phys. Rev. C 60, 064307 (1999).

[10] Y. Kalmykov et al., Phys. Rev. Lett. 96, 012502 (2006). 
[11] H. Aiba, M. Matsuo, S. Nishizaki, and T. Suzuki, Phys. Rev. C 68, 054316 (2003).

[12] P. Ring and P. Schuck, The Nuclear Many-Body Problem (Springer, New York, 1980), Chap. 2.9.

[13] G. F. Bertsch, P. F. Bortignon, and R. A. Broglia, Rev. Mod. Phys. 55, 287 (1983).

[14] S. Drożdż, S. Nishizaki, J. Speth, and J. Wambach, Phys. Rev. C 49, 867 (1994).
[15] G. F. Bertsch, P. F. Bortignon, R. A. Broglia, and C. H. Dasso, Phys. Lett. B 80, 161 (1979).

[16] R. A. Broglia and P. F. Bortignon, Phys. Lett. B 101, 135 (1981).

[17] P. F. Bortignon and R. A. Broglia, Nucl. Phys. A 371, 405 (1981).

[18] D. Lacroix, S. Ayik, and P. Chomaz, Phys. Rev. C 63, 064305 (2001).

[19] R. H. Spear, At. Data Nucl. Data Tables 62, 55 (1989). 